\title{
Review of the future of lung cancer screening
}

\author{
Rowena Yip ${ }^{1}$, Artit Jirapatnakul ${ }^{1}$, Meng Li ${ }^{1,2}$, David F. Yankelevitz ${ }^{1}$, Claudia I. Henschke ${ }^{1}$ \\ ${ }^{1}$ Department of Radiology, Mount Sinai Health System, New York, NY, USA; ${ }^{2}$ Department of Diagnostic Radiology, National Cancer Center/ \\ National Clinical Research Center for Cancer/Cancer Hospital, Chinese Academy of Medical Sciences and Peking Union Medical College, Beijing, \\ China \\ Contributions: (I) Conception and design: R Yip, A Jirapatnakul, DF Yankelevitz, CI Henschke; (II) Administrative support: DF Yankelevitz, CI \\ Henschke; (III) Provision of study materials or patients: R Yip, A Jirapatnakul, DF Yankelevitz, CI Henschke; (IV) Collection and assembly of data: \\ All authors; (V) Data analysis and interpretation: All authors; (VI) Manuscript writing: All authors; (VII) Final approval of manuscript: All authors. \\ Correspondence to: Claudia I. Henschke, PhD, MD. Department of Radiology, One Gustave L. Levy Place, Box 1234, New York, NY 10029, USA. \\ Email: Claudia.Henschke@mountsinai.org.
}

\begin{abstract}
Lung cancer screening has progressed greatly in the last two decades since the initial publication of Early Lung Cancer Action Project (ELCAP) in 1999. Since then, large randomized trials, the National Lung Screening Trial (NLST) and NELSON studies, have also shown the benefit of lung cancer screening with CT, and it is now widely available. There are many areas for improving the benefit of screening by refining the eligibility criteria, continued optimization of the screening regimen, identifying other related diseases, and improved treatment. As artificial intelligence (AI) techniques continue to improve, an automated report of all diseases can be produced from the low-dose CT scan. New screening tests are being developed for lung cancer including blood-based tests. There is growing recognition that use of risk factorbased criteria which dichotomize age and pack-years of smoking to select eligible individuals for screening may not be the most efficient and may also lead to increased lung cancer disparity. Continued research will focus on optimal approaches for selecting high risk individuals for lung cancer screening and thus hopefully expand eligibility criteria beyond the current criteria which include only $20 \%$ of lung cancer patients. With the increase in early stage lung cancers identified from lung cancer screening, a key focus of ongoing research is the treatment of these cancers.
\end{abstract}

Keywords: Lung cancer; screening; future

Received: 09 July 2020; Accepted: 25 June 2021; Published: 25 November 2022.

doi: $10.21037 /$ ccts-20-125

View this article at: https://dx.doi.org/10.21037/ccts-20-125

\section{Introduction}

In the twenty years since the authors initial Early Lung Cancer Action Project (ELCAP) report in 1999 (1-3), lowdose computed tomography (LDCT) screening has been implemented throughout the world (4-11). At the same time, a remarkable transformation in medicine is starting, due in part to the advancing knowledge in genetics, mathematical advances, and increased computing power.

LDCT radiation doses are currently below the dose of mammographic studies. Ultra LDCT have radiation doses approaching those of chest X-ray (CXR) and are being used for evaluation of chest and heart diseases (12). New image analytics and statistical techniques have been developed, with even more innovations on the horizon. Future image interpretation will increasingly use computer-aided-diagnostics, already started as early as the 1990's (12). Further developments will continue to improve the assessment of three-dimensional volumetric doubling time (VDT), already introduced in the mid 1990's by ELCAP. As the experience with LDCT continues to accumulate, the future application of LDCT will be to provide a comprehensive "health check," perhaps together with expanded routine blood testing.

Possible near-term advances are:

(I) Expansion of the eligibility criteria for screening 
using risk-based criteria, particularly to women, never smokers exposed to secondhand tobacco smoke, and to people of younger age.

(II) Integration of artificial intelligence (AI) approaches for LDCT interpretation.

(III) Continued optimization of the regimen of screening.

(IV) Understanding the benefit of identification of other early diseases.

(V) Evaluation of new screening tests will emerge.

(VI) New paradigms of treatment for early lung cancers.

Future development of screening will rely on comprehensive management systems that are integrated into hospital electronic medical record systems and also provide for outreach to potential screening participants. The International Early Lung Cancer Action Program (I-ELCAP) used the ELCAP Management System and provided many new insights for further development of screening regimens. Its success demonstrates the usefulness of comprehensive management systems which provide quality assurance of the interpretation and follow-up management needed in screening programs. The ELCAP Management System started in 1992 and has continued to be updated and used throughout the world. Its database has allowed for further development of the LDCT screening regimen and its efficiency as demonstrated by its contributions (12). The ELCAP Management System has now been translated into an open-source management system called VAPALS-ELCAP Management System for the Veterans Administration Health Care System. These large emerging high-quality databases, developed for comprehensive clinical management of screening, will become invaluable for further development of screening for lung cancer.

\section{Expansion of the eligibility criteria for screening using risk-based criteria, particularly to women, never smokers exposed to secondhand tobacco smoke, and to people of younger age}

The National Lung Screening Trial (NLST) (6) and NELSON trial (11) showed that LDCT screening decreases lung cancer mortality rates for current and former smokers when compared with chest radiographic screening or no screening, respectively. The NLST criteria for screening was current and former smokers with at least 30 pack-years of smoking and for the former smokers, only those who had quit in the last 15 years prior to enrollment.

The authors have previously shown that women are at higher risk to develop lung cancer than equally smoking men (13-16). When the probability of lung cancer among I-ELCAP smokers with sex, age, pack-years of smoking and years since quit was modeled a 55 -year-old man with at least 30 pack-years of smoking would have about the same risk as a 47 year-old woman with the same smoking history (17). This result suggested that if the NLST entry criteria used 55 as the age cutoff for men to be eligible for screening, the corresponding age cutoff for women should be lower, at around 47 years old (17). This finding corroborates other studies that women with lung cancer are diagnosed at a younger age than men (18-20). Expanding screening to include women at a lower age would allow identification of those high-risk women who are currently outside of the guidelines. In fact, results of the NELSON trial also support the consideration to extend screening to younger smokers. The NELSON trial (7) included smokers aged 50 and older with a lower smoking history than the NLST, and the trial showed a greater lung cancer mortality reduction when comparing LDCT screening with no screening. Expansion of the NLST criteria is being considered in the United States and elsewhere as it has been estimated that only $20 \%$ of the lung cancer patients diagnosed each year in the United States met these criteria.

Recently, lung cancer in never smokers, particularly in women has attracted attention. Never smokers are defined as having smoked less than 100 cigarettes in their lifetime (21). It is estimated that $10-25 \%$ of lung cancers occur in never-smokers so that lung cancer in never smokers is the 7th most common malignancy resulting in approximately 300,000 deaths annually (22-24). Lung cancer in never smokers has been considered by some to be a "different lung cancer" compared to smoking associated lung cancer $(22,25)$. Epidemiologic studies showed that lung cancer in never smokers occurred more frequently in women $(53 \%)$ and at a relatively young age compared to lung cancer in smokers which is traditionally found in elderly men. Among East Asian women, $61-80 \%$ of the lung cancer diagnoses were in never smokers (26-28). This high frequency is thought to reflect exposures to secondhand tobacco smoke, indoor air pollution from burning of coal for heating and cooking fumes, and outdoor pollution from ambient fine particles $(29,30)$. The most common celltype in never smokers was adenocarcinoma. There are also differences in genetics and molecular findings as these cancers are often associated with epidermal growth factor receptor (EGFR) mutations and anaplastic lymphoma kinase (ALK) rearrangements while smoking related lung 
cancers are associated with KRAS mutations (31-33); these differences can be used to guide specific targeted therapies. In light of the decreasing rates of cigarette smoking, the proportion of lung cancers diagnosed in never smokers is increasing (34).

Expansion of eligibility criteria for individuals who never smoked is becoming an important issue. As eligibility criteria expand, attention to the regimen of screening, and continuous updating of an evidence-based nodule management protocol, is important to limit unnecessary workup and over-treatment. Results from Eastern Asian countries showed that LDCT screening can detect a significant number of lung cancers in never smokers, most of them in early stage; approximately $70 \%$ of these lung cancers would have been missed using the NLST selection criteria for screening (35-37). These studies recommended that individuals who have never smoked should be included in eligibility criteria of LDCT screening. Results in the United States also suggest that LDCT screening is beneficial for never smokers exposed to secondhand tobacco smoke for identification of early lung cancer, cardiovascular disease and emphysema (38). Future studies, with focus on young never smoking women, will be needed to address this issue.

Several validated lung cancer risk prediction models are available for ever-smokers. Earlier risk prediction models for lung cancer that considered never smokers, however, were limited as they were either developed in case-control studies which may produce biased samples (39-42) or the predictive accuracy was poor with area under the receiver operating characteristic curves (AUCs) in the region of $0.5(39,43)$. In the early 2010s, a few risk prediction models were developed to identify high risk individuals in the general population (regardless of smoking history). However, these models only captured the never smoking status by inverting the smoking duration or intensity to zero, or by including an indicator for 'never-smokers' or 'never-smokers with secondhand tobacco smoke exposure' in their model (44-47). Furthermore, they did not consider many of the distinct risk factors which are unique to never smokers and thus failed to capture the entire spectrum of exposure among never smokers. Despite the overall good calibration and discrimination demonstrated by the $\mathrm{PLCO}_{\mathrm{M} 2014}$ model, when model performance was evaluated separately by neverand ever-smokers, results indicated that the $\mathrm{PLCO}_{\mathrm{M} 2014}$ model had much higher discriminatory power in eversmokers than in never smokers (AUC of $0.85-0.86$ for eversmokers vs. AUC of 0.62 for never-smokers) (44). Concerns about misclassification and inaccuracy in the classification of smoking and exposure status have also been raised $(48,49)$, although others have shown reasonable correlations of selfreported smoking status and biologic confirmation (50).

Since lung cancer is traditionally associated with smoking, the risk in never-smokers remains underrecognized. In recent years there are, however, increasing efforts to understand the etiology of lung cancer in never smokers (21,51-56). Most studies have reported links to family history of cancer, genetic susceptibility, occupational exposure, environmental exposure including secondhand tobacco exposure, air-pollution and cooking oil fumes. Some evidence also suggested the effect of diet and lifestyle on risk of lung cancer while the role of hormones remained unclear. Unfortunately, most of these risk factors have been recently identified and were not collected in historical datasets. These analyses require complex assessment of exposure or additional cost and thus have limited progress in the development and validation of risk models for never smokers. With the advancement in technology, big data analytics and increasing awareness of the risk of lung cancer in never smokers, more researchers are now working to develop personalized risk prediction tools to more accurately stratify lung cancer risk across smoking status by integrating clinical, laboratory and biomarkers/genetic information (57-60). Through better risk assessment, the effectiveness of screening can be increased by identifying those never smokers who have as high a risk of developing lung cancer as some heavy smokers.

In the era of precision medicine, there is growing recognition that use of these risk factor-based criteria which dichotomize age and pack-years of smoking to select eligible individuals for screening may not be the most efficient and may also lead to increase lung cancer disparity $(18,61)$. Furthermore, multiple retrospective analyses and modeling studies have demonstrated that risk prediction models that incorporate additional risk factors help improve risk stratifications. These models demonstrated higher sensitivity and positive predictive value, and increased cost-effectiveness than older, less complex criteria for determining screening eligibility (44,62-64). A few studies are currently underway to prospectively evaluate the use of risk prediction models and to determine the optimal approach for selecting high risk individuals for lung cancer screening (65-68). Despite the superiority over risk factorbased criteria in preventing lung cancer deaths, concerns have been raised that risk prediction models may be suboptimal and increase overdiagnosis. As these lung cancer 
risk prediction models do not account for life-expectancy, they are more likely to select older smokers with longer smoking history and more comorbid conditions. These individuals are more likely to die from competing causes and may not live long enough to experience the net benefit of screening. To address these issues, alternative models that select individuals based on life-years gained have also been proposed (69-71).

\section{Integration of Al approaches for LDCT interpretation}

AI techniques in the field of medicine are gaining more interest recently, but it should be recognized that initial efforts had started as early as in 1970s (12). Due to enhanced algorithms and computing power, AI has made great strides in many areas - classification of photos, person recognition, self-driving vehicles, natural language processing, and data mining, just to name a few and has generated great enthusiasm for streamlining cancer screening to improve early detection and diagnosis of cancer and personalize treatment and outcome prediction (72-78). The future of AI in lung cancer screening lies in the integration of algorithms that detect and diagnose all diseases visible in a LDCT, not only lung cancer but emphysema, interstitial lung disease, cardiovascular disease, and liver disease to automatically produce a report of everything that is visible on a LDCT chest scan.

AI algorithms for lung cancer screening have focused on the detection and characterization of pulmonary nodules. Early studies used manually engineered features, where an algorithm is designed to compute a specific feature, such as the distribution of density within the nodule, and these studies either targeted the detection of nodules $(79,80)$ or the classification of nodules as benign or malignant $(81,82)$. However, performance of these systems is still not sufficient for completely automated use. Nodule detection results are commonly reported as the sensitivity, the rate of detecting actual nodules, for a given number of false positives, detection of objects that are not nodules. Recent comparative studies of research nodule detection systems report results in the range of $75-85 \%$ sensitivity with an average of a single false positive per case $(83,84)$. Likewise, the performance of nodule classification systems still leaves much to be desired, with reported AUC performance of 0.75 . Although these results are not sufficiently high for a completely automated system, software systems are now available from many commercial vendors that include tools for nodule detection, measurement, and classification that are intended to help support radiologists' interpretation.

As a result of recent successes of neural networks and deep learning in the computer vision area, in particular the breakthrough performance deep learning on the ImageNet challenge (85), a competition to classify images into 1,000 different categories, deep learning has been applied to all areas of image interpretation (86). A team of researchers at Google applied deep learning to detecting diabetic retinopathy from retinal fundus photographs with great success-they achieved a performance of over $90 \%$ sensitivity and specificity (87). Another recent study developed a deep learning algorithm to predict the risk of lung cancer (88). The authors report an AUC of 0.944 for predicting lung cancer, performing as well as or better than radiologists. However, the system was developed and tested on data from the NLST, which was collected over 15 years ago; CT technology has made great strides during this time, and the high performance on the NLST dataset may not translate to modern images. In addition to lung cancer $(89,90)$, deep learning has also been applied to quantification of aortic calcification (91), emphysema (92), and breast cancer $(93,94)$.

As AI continues to improve, there are two technical obstacles to overcome. Deep learning, more so than previous AI approaches, requires large amounts of data; however, there is a lack of large, public, well-documented databases of medical images. A key reason for the success of computer vision in the ImageNet challenge is its database which allows research groups all over the world to train and test their algorithm on the same database as others in the field, allowing for direct comparisons between algorithms. Unfortunately, while there have been efforts to create such databases for lung screening, such as the public lung database (PLD) (95), NLST (96), ANODE09 (97), LUNA16 (98), and The Cancer Imaging Archive (TCIA) (99) to name a few, these all have few cases-from 50 to a couple thousand. As a point of comparison, the ImageNet database contains over 1 million images. There are many reasons for the lack of such large public databases-patient privacy, funding, and time and difficulty in properly annotating data to name a few.

A second major technical obstacle facing the continued improvement of AI is the lack of consistency between different scanners and protocols in the images that are acquired. This issue has been recognized (100), with studies showing that radiomic features have low reproducibility $(101,102)$, and that CT reconstruction parameters can 
affect the performance of radiomics systems (103). There is ongoing work in developing tools and methods for characterizing CT scanner performance (104), so that in the future, radiomic features and quantitative measurements can be consistent across scanners and acquisition protocols.

In conclusion, the field is rapidly progressing towards the goal of automatically producing a report of everything that is visible in the lung, not only related to lung cancer, but also other diseases. The individual algorithms exist today, but much work remains to improve their performance. This will require large, documented databases and high-quality imaging.

\section{Continued optimization of the regimen of screening}

Since 1992, when the initial regimen of screening was developed, it was recognized that continual updating was needed to integrate technologic and knowledge advances (12). Growth assessment was an important component to differentiate between lung cancer and benign nodules. This led to recognition of the measurement errors of CT scanners. The need for large data and image repositories was also recognized, particularly in light of the new statistical approaches using AI techniques. Risk models are being developed to provide follow-up recommendations. Lessons learned in the past years since the introduction of LDCT screening studies starting in 1992 (12) are useful in illustrating the importance of accumulated knowledge and data to guide approaches in the future.

When assessing the benefit of screening, it is important to understand implications of the trial designs in assessing the benefit of screening (105-107). This is particularly important when providing information about the benefits and risks of screening to a participant seeking the screening $(108,109)$. The high cure rates of small, early, screeningdiagnosed lung cancers are frequently not presented. It needs to be recognized that the lung-cancer mortality reductions of randomized screening trials do not present the benefit of an early diagnosis which is the cure rate of screening-diagnosed lung cancers (108). It needs to be understood that the NLST and NELSON trials were designed to show a mortality reduction of $20 \%$ and $25 \%$, respectively to justify screening. These two trials were not designed to provide the cure rate of screen-diagnosed lung cancers. Future development of better tools to explain the benefit of LDCT, or any other screening test for any cancer, should be developed. Increasing patients' and providers' awareness of the benefit of screening is key to improving both uptake and adherence to lung cancer screening.

Approaches for comparisons of different regimens of screening are needed to determine the optimal work-up for nodules detected on baseline and annual repeat rounds of screening, by nodule consistency. One such approach was developed to compare the International Early Lung Cancer Action Protocol with those of the American College of Radiology LungRADS and the European Protocol (110). Continuous update of protocols to incorporate advancing technology and knowledge will minimize unnecessary diagnostic work-ups and biopsies/surgeries. Research is being performed to identify new predictors of benign and malignant/aggressive etiology, especially with the help of AI. These include perifissural and costal-pleural nodules (111).

The regimen should strive to maximize the likelihood of early diagnosis of lung cancer while minimizing unnecessary invasive workup. The importance of having a well-defined regimen was demonstrated by the comparison of I-ELCAP results with those of the NLST which did not specify a regimen. I-ELCAP's higher percentage of Stage I diagnoses and long-term survival rates compared with those of the NLST, after consideration of multiple alternative explanations, was due to the I-ELCAP regimen (112).

Aside from a well-defined regimen, appropriate radiologic interpretation can minimize unnecessary workup and interventional procedures. It has also been shown that high quality LDCT screening can be performed in academic or community settings as long as a quality assurance process is in place (113). Integration of new image analytic tools being developed should further improve the diagnostic interpretation and lead to a reduction of unnecessary further testing and reduce the frequency of surgical resection of benign nodules. It has been shown that, by carefully following a well-defined regimen of screening, the frequency of benign resection can be below $5 \%$ (114).

Volumetric measurement is acknowledged to be a better assessment of nodule size. Volume doubling time is a more reliable measure of growth for distinguish between malignant and non-malignant nodules. However, volumetric measurements need to be interpreted cautiously with regard to CT acquisition parameters and CT measurement errors (115). The recent update of Lung-RADS version 1.1 added volume measurements next to the nodule diameter measurements. Use of volume measurement to provide more accurate growth assessment over time is expected to increase as well as further refinement of growth assessment, 
including use of phantoms to adjust for measurement variability of the CT will improve measurement accuracy. The ability to reliably assess tumor growth and advancement in prediction tools can help inform follow-up interval/screening interval (116).

Development of blood-based tests will continue to develop and, when proven to be useful, should be integrated into the screening process. New pathologic criteria will continue to emerge together with advances in lung cancer biomarkers as has already been demonstrated by the revised World Health Organization classifications (117-120). In the future, more precise biologic information as to the aggressiveness of the screening-diagnosed lung cancers will be achieved by improved imaging of growth in a timely manner and pathologic biomarkers.

\section{Understanding the benefit of identification of other early diseases identified}

It has been recognized that LDCT screening provides a comprehensive "health check" of the lungs, heart, and other organs visualized on the LDCT. This vision is gaining increasing recognition throughout the world. An entire session at the $20^{\text {th }}$ World Conference on Lung Cancer (WCLC) in Barcelona, Spain in September 2019 was devoted to these other findings (121). I-ELCAP protocol recommendations for these other findings were developed together with relevant medical specialties, with the recognition that these are findings in asymptomatic screening participants and not patients presenting to physicians with symptoms. Initial focus was on the cardiac findings $(122,123)$ and emphysema (124-126) which, together with lung cancer, are the three big killers of older smokers. This focus has now been expanded to consider the other findings on the LDCT of the chest and the recommendations are provided in the I-ELCAP protocol (127).

Such a comprehensive "health check" optimal LDCT screening requires a carefully-specified, validated regimen which provides for identification and interpretation of critical LDCT findings and the appropriate follow-up recommendations. The recommendations need to be developed together with the relevant medical specialties and may differ in different medical care settings.

\section{Evaluation of new screening tests will emerge}

As new screening tests emerge, both imaging and bloodbased ones, it should be recognized that these new tests can be rapidly evaluated using designs similar to the low-cost, efficient prospective ELCAP cohort design. Two rounds of screening, a baseline round and a single annual repeat round can provided pertinent information on the tumor size at detection, the stage shift $(1,2)$, and after appropriate follow-up, the cure rates (3). This same design can be used to test new blood tests for earlier detection of lung cancer. Both the blood test and a LDCT can be given to individuals at risk of lung cancer and tested within two years.

Hopefully there will be recognition that randomized start-stop trials to test methods of screening for a cancer do not provide the ultimate benefit of the screening test, but rather only provide that a minimum level of benefit is met before providing the screening on a larger populationwide basis. For example the NLST required 3 rounds of screening with 5.5 years of follow-up to show at least a $20 \%$ lung cancer-specific mortality reduction by LDCT screening compared to chest radiography; its results took nine years from start to publication (6). The NELSON Trial, started in 2004 to provide 4 rounds of screening with 10 years of follow-up (11), reported that it provided at least a $25 \%$ lung cancer-specific mortality reduction compared to no screening.

\section{New paradigms of treatment for early lung cancers}

As already seen in the past 20 years since screening was introduced, understanding of the pathologic findings of small, early lung cancers has increased. Identification of small, early cancers has stimulated advances in treatment of early lung cancer which in turn has led to updates in the pathologic and staging criteria. The current recommendation of lobar resection of Stage I lung cancers has not changed in more than 50 years.

The authors postulate that, similar to the impact of breast cancer screening which transformed treatment of breast cancer from radical mastectomy to a very nuanced, personalized approach, lung cancer treatment will become more personalized with an emphasis on post-treatment quality of life and preservation of lung tissue as new primary lung cancers may emerge.

LDCT screening has already led to significant changes in the pathologic classification and the $8^{\text {th }}$ Staging Classification (128-130). Screening results stimulated two randomized surgical trials comparing lobectomy with sublobar resection which started in 2007, one in Japan and the other in the United States $(131,132)$. Both anticipate 
publishing their final results around 2020 and their interim reports are encouraging as both have shown extremely low rates of surgical deaths $(133,134)$.

New technologies have been introduced such as robotic surgery, navigational bronchoscopy, ablation approaches, and in the future, there will be further innovations. None of these, however, have had critical assessment, and often there is only limited data for small lung cancers. Published cohort studies using the I-ELCAP database (114,135-138) have already provided timely outcome results and Quality of Life measures which will become an increasingly important consideration in treatment determination given the high long-term cure rates of screen-diagnosed lung cancer (139-143).

For timely assessment of new treatments, the Initiative for Early Lung Cancer Research on Treatment (IELCART) (144) was started using the same prospective cohort design used for I-ELCAP and capturing treatment information in the context of clinical care. The ELCAP Management System, used for both management and research purposes allowed for the accumulation of over 82,000 participants with clinical data, imaging and biologic specimens, has been adapted for the multi-institutional, international IELCART database. The system also allows for randomization for future innovative randomized trials. The vision for IELCART is to become as productive as I-ELCAP has been in producing ongoing screening evidence.

\section{Conclusions}

Continued advances in CT technology, including reduction in radiation dose as well as new image analytics and statistical techniques being developed will continue to improve LDCT screening, perhaps together with new pre- and post-CT tests, such as those currently under development. Optimizing the screening will reduce the frequency of unnecessary workup and invasive diagnostics, increase the frequency of earlier diagnoses, and provide for an even more comprehensive "health check." In light of the continued advances in screening for lung cancer and the integration of the advances in screening protocols, a key focus of ongoing and future research should be on the treatment of these cancers.

\section{Acknowledgments}

Funding: None.

\section{Footnote}

Provenance and Peer Review: This article was commissioned by the Guest Editors (Scott Swanson, Daniel Dolan) for the series "How to Evaluate, Diagnose and Treat Small Lung Nodules" published in Current Challenges in Thoracic Surgery. The article has undergone external peer review.

Conflicts of Interest: All authors have completed the ICMJE uniform disclosure form (available at https:// ccts.amegroups.com/article/view/10.21037/ccts-20-125/ coif). The series "How to Evaluate, Diagnose and Treat Small Lung Nodules" was commissioned by the editorial office without any funding or sponsorship. DFY is a consultant and co-owner of Accumetra, a private company developing tools to improve the quality of CT imaging, and he is on the medical advisory board of Carestream, a company that develops radiography equipment, and he has consulted for Genentech, AstraZeneca and Pfizer. In addition, DFY is a named inventor on a number of patents and patent applications related to the evaluation of chest diseases including measurements of chest nodules. DFY has received financial compensation for the licensing of these patents. $\mathrm{CH}$ is a named inventor on a number of patents and patent applications relating to the evaluation of pulmonary nodules on CT scans of the chest which are owned by Cornell Research Foundation (CRF). Since 2009, CH does not accept any financial benefit from these patents including royalties and any other proceeds related to the patents or patent applications owned by CRF. CH is the President and serve on the board of the Early Diagnosis and Treatment Research Foundation. $\mathrm{CIH}$ receives no compensation from the Foundation. The Foundation is established to provide grants for projects, conferences, and public databases for research on early diagnosis and treatment of diseases. Recipients include, I-ELCAP, among others. The funding comes from a variety of sources including philanthropic donations, grants and contracts with agencies (federal and nonfederal), imaging and pharmaceutical companies relating to image processing assessments. The various sources of funding exclude any funding from tobacco companies or tobacco-related sources. The authors have no other conflicts of interest to declare.

Ethical Statement: The authors are accountable for all aspects of the work in ensuring that questions related to the accuracy or integrity of any part of the work are 
appropriately investigated and resolved.

Open Access Statement: This is an Open Access article distributed in accordance with the Creative Commons Attribution-NonCommercial-NoDerivs 4.0 International License (CC BY-NC-ND 4.0), which permits the noncommercial replication and distribution of the article with the strict proviso that no changes or edits are made and the original work is properly cited (including links to both the formal publication through the relevant DOI and the license). See: https://creativecommons.org/licenses/by-nc-nd/4.0/.

\section{References}

1. Henschke CI, McCauley DI, Yankelevitz DF, et al. Early Lung Cancer Action Project: overall design and findings from baseline screening. Lancet 1999;354:99-105.

2. Henschke CI, Naidich DP, Yankelevitz DF, et al. Early lung cancer action project: initial findings on repeat screenings. Cancer 2001;92:153-9.

3. International Early Lung Cancer Action Program Investigators; Henschke CI, Yankelevitz DF, et al. Survival of patients with stage I lung cancer detected on CT screening. N Engl J Med 2006;355:1763-71.

4. Centers of Medicare and Medicaid Services. Decision memo for screening for lung cancer with low dose computed tomography (LDCT) (CAG-00439N). Available online: http://www.cms.gov/medicare-coverage-database/ details/nca-decision-memo.aspx?NCAId.274

5. Chong S, Lee KS, Chung MJ, et al. Lung cancer screening with low-dose helical CT in Korea: experiences at the Samsung Medical Center. J Korean Med Sci 2005;20:402-8.

6. National Lung Screening Trial Research Team; Aberle DR, Adams AM, et al. Reduced lung-cancer mortality with low-dose computed tomographic screening. $\mathrm{N}$ Engl J Med 2011;365:395-409.

7. De Koning H, Van der Aalst C, Ten Haaf K, et al. Effects of Volume CT Lung Cancer Screening: Mortality Results of the NELSON Randomised-Controlled Population Based Trial. J Thorac Oncol 2018;13:S185.

8. Pastorino U, Silva M, Sestini S, et al. Prolonged lung cancer screening reduced 10-year mortality in the MILD trial: new confirmation of lung cancer screening efficacy. Ann Oncol 2019;30:1162-9.

9. Pastorino U, Rossi M, Rosato V, et al. Annual or biennial CT screening versus observation in heavy smokers: 5-year results of the MILD trial. Eur J Cancer Prev
2012;21:308-15.

10. Triphuridet N, Henschke C. Landscape on CT screening for lung cancer in Asia. Lung Cancer (Auckl) 2019;10:107-24.

11. de Koning HJ, van der Aalst CM, de Jong PA, et al. Reduced Lung-Cancer Mortality with Volume CT Screening in a Randomized Trial. N Engl J Med 2020;382:503-13.

12. Henschke CI, Yip R, Shaham D, et al. The Regimen of Computed Tomography Screening for Lung Cancer: Lessons Learned Over 25 Years From the International Early Lung Cancer Action Program. J Thorac Imaging 2021;36:6-23.

13. Henschke CI, Miettinen OS. Women's susceptibility to tobacco carcinogens. Lung Cancer 2004;43:1-5.

14. International Early Lung Cancer Action Program Investigators; Henschke CI, Yip R, et al. Women's susceptibility to tobacco carcinogens and survival after diagnosis of lung cancer. JAMA 2006;296:180-4.

15. Yip R, Fevrier E, Wolf A, et al. Lung cancer: susceptibility and survival differences for women and men. J Thorac Oncol 2019;14:S527.

16. Henschke C. Lung Screening for Women-Susceptibility and Cure Rates 2019. Available online: https://www.iaslc. org/Conferences-Events/Event-Details/women-and-lungcancer-screening-treatment-and-beyond

17. I-ELCAP Investigators. Optimal age eligibility criteria for low-dose screening for lung cancer in women. 2019. Available online: https://www.iaslc.org/meetings-webinars/ women-and-lung-cancer-screening-treatment-and-beyond

18. Pasquinelli M, Kovitz K, Durham M, et al. Expanding Criteria for Lung Cancer Screening Reduces Gender Disparity. J Thorac Oncol 2019;14:S517.

19. Jemal A, Miller KD, Ma J, et al. Higher Lung Cancer Incidence in Young Women Than Young Men in the United States. N Engl J Med 2018;378:1999-2009.

20. Fidler-Benaoudia MM, Torre LA, Bray F, et al. Lung cancer incidence in young women vs. young men: A systematic analysis in 40 countries. Int $\mathrm{J}$ Cancer 2020;147:811-9.

21. Couraud S, Zalcman G, Milleron B, et al. Lung cancer in never smokers--a review. Eur J Cancer 2012;48:1299-311.

22. Sun S, Schiller JH, Gazdar AF. Lung cancer in never smokers--a different disease. Nat Rev Cancer 2007;7:778-90.

23. Jemal A, Bray F, Center MM, et al. Global cancer statistics. CA Cancer J Clin 2011;61:69-90.

24. Torok S, Hegedus B, Laszlo V, et al. Lung cancer in never 
smokers. Future Oncol 2011;7:1195-211.

25. Smolle E, Pichler M. Non-Smoking-Associated Lung Cancer: A distinct Entity in Terms of Tumor Biology, Patient Characteristics and Impact of Hereditary Cancer Predisposition. Cancers (Basel) 2019;11:204.

26. Khuder SA. Effect of cigarette smoking on major histological types of lung cancer: a meta-analysis. Lung Cancer 2001;31:139-48.

27. Parkin DM, Bray F, Ferlay J, et al. Global cancer statistics, 2002. CA Cancer J Clin 2005;55:74-108.

28. Emery JD, Mitchell PL. Lung cancer in Asian women and health system implications for Australia. Lancet Oncol 2017;18:1570-1.

29. Chen W, Zheng R, Zhang S, et al. Annual report on status of cancer in China, 2010. Chin J Cancer Res 2014;26:48-58.

30. Bray F, Ferlay J, Soerjomataram I, et al. Global cancer statistics 2018: GLOBOCAN estimates of incidence and mortality worldwide for 36 cancers in 185 countries. CA Cancer J Clin 2018;68:394-424.

31. Subramanian J, Govindan R. Molecular genetics of lung cancer in people who have never smoked. Lancet Oncol 2008;9:676-82.

32. Subramanian J, Govindan R. Molecular profile of lung cancer in never smokers. EJC Suppl 2013;11:248-53.

33. Chen YJ, Roumeliotis TI, Chang YH, et al. Proteogenomics of Non-smoking Lung Cancer in East Asia Delineates Molecular Signatures of Pathogenesis and Progression. Cell 2020;182:226-244.e17.

34. Pelosof L, Ahn C, Gao A, et al. Proportion of NeverSmoker Non-Small Cell Lung Cancer Patients at Three Diverse Institutions. J Natl Cancer Inst 2017. doi: 10.1093/jnci/djw295.

35. Kakinuma R, Muramatsu $\mathrm{Y}$, Asamura $\mathrm{H}$, et al. Low-dose CT lung cancer screening in never-smokers and smokers: results of an eight-year observational study. Transl Lung Cancer Res 2020;9:10-22.

36. Zhang Y, Jheon S, Li H, et al. Results of low-dose computed tomography as a regular health examination among Chinese hospital employees. J Thorac Cardiovasc Surg 2020;160:824-831.e4.

37. Kang HR, Cho JY, Lee SH, et al. Role of Low-Dose Computerized Tomography in Lung Cancer Screening among Never-Smokers. J Thorac Oncol 2019;14:436-44.

38. Henschke C, Yip R, Yankelevitz D. P2.11-08 CT Screening of Never Smokers. J Thorac Oncol 2019;14:S794-5.

39. Spitz MR, Hong WK, Amos CI, et al. A risk model for prediction of lung cancer. J Natl Cancer Inst
2007;99:715-26.

40. Cassidy A, Myles JP, van Tongeren M, et al. The LLP risk model: an individual risk prediction model for lung cancer. Br J Cancer 2008;98:270-6.

41. Etzel CJ, Kachroo S, Liu M, et al. Development and validation of a lung cancer risk prediction model for AfricanAmericans. Cancer Prev Res (Phila) 2008;1:255-65.

42. Kovalchik SA, Tammemagi M, Berg CD, et al. Targeting of low-dose CT screening according to the risk of lungcancer death. N Engl J Med 2013;369:245-54.

43. Hoggart C, Brennan P, Tjonneland A, et al. A risk model for lung cancer incidence. Cancer Prev Res (Phila) 2012;5:834-46.

44. Tammemägi MC, Church TR, Hocking WG, et al. Evaluation of the lung cancer risks at which to screen everand never-smokers: screening rules applied to the PLCO and NLST cohorts. PLoS Med 2014;11:e1001764.

45. Marcus MW, Chen Y, Raji OY, et al. LLPi: Liverpool Lung Project Risk Prediction Model for Lung Cancer Incidence. Cancer Prev Res (Phila) 2015;8:570-5.

46. Hippisley-Cox J, Coupland C. Development and validation of risk prediction algorithms to estimate future risk of common cancers in men and women: prospective cohort study. BMJ Open 2015;5:e007825.

47. Charvat H, Sasazuki S, Shimazu T, et al. Development of a risk prediction model for lung cancer: The Japan Public Health Center-based Prospective Study. Cancer Sci 2018;109:854-62.

48. Lee PN. Misclassification of smoking habits and passive smoking: a review of the evidence: Springer Science \& Business Media, 2013.

49. The Health Consequences of Involuntary Exposure to Tobacco Smoke: A Report of the Surgeon General. Atlanta (GA): Publications and Reports of the Surgeon General. 2006.

50. Liu B, Henschke CI, Flores RM, et al. Serum cotinine verification of self-reported smoking status among adults eligible for lung cancer screening in the 1999-2018 National Health and Nutrition Examination Survey. Lung Cancer 2020;144:49-56.

51. Samet JM, Avila-Tang E, Boffetta P, et al. Lung cancer in never smokers: clinical epidemiology and environmental risk factors. Clin Cancer Res 2009;15:5626-45.

52. Gorlova OY, Weng SF, Hernandez L, et al. Dietary patterns affect lung cancer risk in never smokers. Nutr Cancer 2011;63:842-9.

53. Pallis AG, Syrigos KN. Lung cancer in never smokers: disease characteristics and risk factors. Crit Rev Oncol 
Hematol 2013;88:494-503.

54. Dias M, Linhas R, Campainha S, et al. Lung cancer in never-smokers - what are the differences? Acta Oncol 2017;56:931-5.

55. Bhopal A, Peake MD, Gilligan D, et al. Lung cancer in never-smokers: a hidden disease. J R Soc Med 2019;112:269-71.

56. Corrales L, Rosell R, Cardona AF, et al. Lung cancer in never smokers: The role of different risk factors other than tobacco smoking. Crit Rev Oncol Hematol 2020;148:102895.

57. Wu X, Wen CP, Ye Y, et al. Personalized Risk Assessment in Never, Light, and Heavy Smokers in a prospective cohort in Taiwan. Sci Rep 2016;6:36482.

58. Qian DC, Han Y, Byun J, et al. A Novel PathwayBased Approach Improves Lung Cancer Risk Prediction Using Germline Genetic Variations. Cancer Epidemiol Biomarkers Prev 2016;25:1208-15.

59. Chien LH, Chen CH, Chen TY, et al. Predicting Lung Cancer Occurrence in Never-Smoking Females in Asia: TNSF-SQ, a Prediction Model. Cancer Epidemiol Biomarkers Prev 2020;29:452-9.

60. Wang X, Zhang Y, Hao S, et al. Prediction of the 1-Year Risk of Incident Lung Cancer: Prospective Study Using Electronic Health Records from the State of Maine. J Med Internet Res 2019;21:e13260.

61. Pasquinelli MM, Tammemägi MC, Kovitz KL, et al. Risk Prediction Model Versus United States Preventive Services Task Force Lung Cancer Screening Eligibility Criteria: Reducing Race Disparities. J Thorac Oncol 2020;15:1738-47.

62. Li K, Hüsing A, Sookthai D, et al. Selecting High-Risk Individuals for Lung Cancer Screening: A Prospective Evaluation of Existing Risk Models and Eligibility Criteria in the German EPIC Cohort. Cancer Prev Res (Phila) 2015;8:777-85.

63. Kumar V, Cohen JT, van Klaveren D, et al. Risk-Targeted Lung Cancer Screening: A Cost-Effectiveness Analysis. Ann Intern Med 2018;168:161-9.

64. Cancer Intervention and Surveillance Modeling Network (CISNET) Lung Cancer Working Group, Meza R, Jeon J, et al. Technical Report. Evaluation of the Benefits and Harms of Lung Cancer Screening With Low-Dose Computed Tomography: A Collaborative Modelling Study for the U.S. Preventive Serevics Task Force. In: U.S. Department of Health and Human Services. Agency for Healthcare Research and Quality. 2020.

65. Lim KP, Manners D, Adler B, et al. The LungScreen
WA Project: Feasibility of LDCT Screening with the PLCOm2012 Risk Model and PanCan Nodule Risk Calculator. J Thorac Oncol 2017;12:S2165-6.

66. Balata H, Crosbie P, Barber P, et al. M13 Manchester lung cancer screening, targeting high-risk individuals in deprived areas of the community: results from the first incidence round of screening (1 year). Thorax 2018;73:A253.

67. Lam S, Myers R, Ruparel M, et al. Lung Cancer Screenee Selection by USPSTF Versus PLCOm2012 Criteria Interim ILST Findings. J Thorac Oncol 2019;14:S4-5.

68. Crosbie PA, Balata H, Evison M, et al. Implementing lung cancer screening: baseline results from a communitybased 'Lung Health Check' pilot in deprived areas of Manchester. Thorax 2019;74:405-9.

69. Caverly TJ, Cao P, Hayward RA, et al. Identifying Patients for Whom Lung Cancer Screening Is PreferenceSensitive: A Microsimulation Study. Ann Intern Med 2018;169:1-9.

70. Cheung LC, Berg CD, Castle PE, et al. Life-GainedBased Versus Risk-Based Selection of Smokers for Lung Cancer Screening. Ann Intern Med 2019;171:623-32.

71. Ten Haaf K, Bastani M, Cao P, et al. A Comparative Modeling Analysis of Risk-Based Lung Cancer Screening Strategies. J Natl Cancer Inst 2020;112:466-79.

72. Liu S, Xie Y, Jirapatnakul A, et al. Pulmonary nodule classification in lung cancer screening with threedimensional convolutional neural networks. J Med Imaging (Bellingham) 2017;4:041308.

73. Lee JH, Jirapatnakul AC, Yip R, et al. Using Deep Learning to Predict Emphysema in Early Lung Cancer Screening Low-Dose CT Scan. 104th Scientific Assembly and Annual Meeting, Radiological Society of North America 2018. Chicago, IL; 2018.

74. Fuhrman JD, Crosby J, Yip R, et al. Detection and classification of coronary artery calcifications in low dose thoracic CT using deep learning. Medical Imaging 2019: Computer-Aided Diagnosis; International Society for Optics and Photonics, 2019.

75. Patel D, Tibrewala R, Vega A, et al. Circumventing the solution of inverse problems in mechanics through deep learning: Application to elasticity imaging. Comput Methods Appl Mech Eng 2019;353:448-66.

76. Kann BH, Thompson R, Thomas CR Jr, et al. Artificial Intelligence in Oncology: Current Applications and Future Directions. Oncology (Williston Park) 2019;33:46-53.

77. Goldenberg SL, Nir G, Salcudean SE. A new era: artificial intelligence and machine learning in prostate cancer. Nat 
Rev Urol 2019;16:391-403.

78. Bi WL, Hosny A, Schabath MB, et al. Artificial intelligence in cancer imaging: Clinical challenges and applications. CA Cancer J Clin 2019;69:127-57.

79. Enquobahrie AA, Reeves AP, Yankelevitz DF, et al. Automated detection of small pulmonary nodules in whole lung CT scans. Acad Radiol 2007;14:579-93.

80. Tan M, Deklerck R, Jansen B, et al. A novel computeraided lung nodule detection system for CT images. Med Phys 2011;38:5630-45.

81. Lee MC, Boroczky L, Sungur-Stasik K, et al. Computeraided diagnosis of pulmonary nodules using a two-step approach for feature selection and classifier ensemble construction. Artif Intell Med 2010;50:43-53.

82. Reeves AP, Xie Y, Jirapatnakul A. Automated pulmonary nodule CT image characterization in lung cancer screening. Int J Comput Assist Radiol Surg 2016;11:73-88.

83. Jacobs C, van Rikxoort EM, Murphy K, et al. Computeraided detection of pulmonary nodules: a comparative study using the public LIDC/IDRI database. Eur Radiol 2016;26:2139-47.

84. Setio AAA, Traverso A, de Bel T, et al. Validation, comparison, and combination of algorithms for automatic detection of pulmonary nodules in computed tomography images: The LUNA16 challenge. Med Image Anal 2017;42:1-13.

85. Krizhevsky A, Sutskever I, Hinton GE. ImageNet Classification with Deep Convolutional Neural Networks. Adv Neural Inf Process Syst 2012:1097-105.

86. Afshar P, Mohammadi A, Plataniotis KN, et al. From Handcrafted to Deep-Learning-Based Cancer Radiomics: Challenges and opportunities. IEEE Signal Process Mag 2019;36:132-60.

87. Gulshan V, Peng L, Coram M, et al. Development and Validation of a Deep Learning Algorithm for Detection of Diabetic Retinopathy in Retinal Fundus Photographs. JAMA 2016;316:2402-10.

88. Ardila D, Kiraly AP, Bharadwaj S, et al. End-to-end lung cancer screening with three-dimensional deep learning on low-dose chest computed tomography. Nat Med 2019;25:954-61.

89. Ciompi F, Chung K, van Riel SJ, et al. Towards automatic pulmonary nodule management in lung cancer screening with deep learning. Sci Rep 2017;7:46479.

90. Hosny A, Parmar C, Coroller TP, et al. Deep learning for lung cancer prognostication: A retrospective multi-cohort radiomics study. PLoS Med 2018;15:e1002711.

91. Graffy PM, Liu J, O'Connor S, et al. Automated segmentation and quantification of aortic calcification at abdominal CT: application of a deep learning-based algorithm to a longitudinal screening cohort. Abdom Radiol (NY) 2019;44:2921-8.

92. Humphries SM, Notary AM, Centeno JP, et al. Deep Learning Enables Automatic Classification of Emphysema Pattern at CT. Radiology 2020;294:434-44.

93. Kallenberg M, Petersen K, Nielsen M, et al. Unsupervised Deep Learning Applied to Breast Density Segmentation and Mammographic Risk Scoring. IEEE Trans Med Imaging 2016;35:1322-31.

94. Li H, Giger ML, Huynh BQ, et al. Deep learning in breast cancer risk assessment: evaluation of convolutional neural networks on a clinical dataset of full-field digital mammograms. J Med Imaging (Bellingham) 2017;4:041304.

95. Reeves AP, Biancardi AM, Yankelevitz D, et al. A public image database to support research in computer aided diagnosis. Annu Int Conf IEEE Eng Med Biol Soc 2009;2009:3715-8.

96. National Lung Screening Trial Research Team; Aberle DR, Berg CD, et al. The National Lung Screening Trial: overview and study design. Radiology 2011;258:243-53.

97. van Ginneken B, Armato SG 3rd, de Hoop B, et al. Comparing and combining algorithms for computeraided detection of pulmonary nodules in computed tomography scans: The ANODE09 study. Med Image Anal 2010;14:707-22.

98. Jacobs C, Setio AAA, Traverso A, et al. LUng Nodule Analysis 2016 2016. Available online: https://luna16.grandchallenge.org/

99. The Cancer Imaging Archive 2020. Available online: https://www.cancerimagingarchive.net/

100. Rydzak CE, Armato SG, Avila RS, et al. Quality assurance and quantitative imaging biomarkers in low-dose CT lung cancer screening. Br J Radiol 2018;91:20170401.

101.Perrin T, Midya A, Yamashita R, et al. Short-term reproducibility of radiomic features in liver parenchyma and liver malignancies on contrast-enhanced CT imaging. Abdom Radiol (NY) 2018;43:3271-8.

102. Talwar A, Willaime JMY, Pickup LC, et al. Pulmonary nodules: Assessing the imaging biomarkers of malignancy in a "coffee-break". Eur J Radiol 2018;101:82-6.

103. Kim H, Park CM, Gwak J, et al. Effect of CT Reconstruction Algorithm on the Diagnostic Performance of Radiomics Models: A Task-Based Approach for Pulmonary Subsolid Nodules. AJR Am J Roentgenol 2019;212:505-12. 
104.Avila R, Henschke C, Yankelevitz D. Quality Control for Lung Imaging Biomarkers. J Thorac Oncol 2017;12:S1743.

105. International Early Lung Cancer Program Investigators. An update of CT screening for lung cancer. Semin Ultrasound CT MR 2005;26:348-56.

106. Henschke CI, Boffetta P, Gorlova O, et al. Assessment of lung-cancer mortality reduction from CT Screening. Lung Cancer 2011;71:328-32.

107.Foy M, Yip R, Chen X, et al. Modeling the mortality reduction due to computed tomography screening for lung cancer. Cancer 2011;117:2703-8.

108. Yankelevitz DF. CT Screening for Lung Cancer: Successful Trial, but Failed Understanding. J Thorac Oncol 2018;13:12-5.

109. Yankelevitz DF, Chan C, Henschke CI. Overdiagnosis: "A Malformed Concept". J Thorac Imaging 2019;34:151-3.

110.Henschke CI, Yip R, Ma T, et al. CT screening for lung cancer: comparison of three baseline screening protocols. Eur Radiol 2019;29:5217-26.

111.Zhu Y, Yip R, You N, et al. Management of Nodules Attached to the Costal Pleura at Low-Dose CT Screening for Lung Cancer. Radiology 2020;297:710-8.

112. Yip R, Henschke CI, Yankelevitz DF, et al. The impact of the regimen of screening on lung cancer cure: a comparison of I-ELCAP and NLST. Eur J Cancer Prev 2015;24:201-8.

113.Xu DM, Lee IJ, Zhao S, et al. CT screening for lung cancer: value of expert review of initial baseline screenings. AJR Am J Roentgenol 2015;204:281-6.

114. Flores R, Bauer T, Aye R, et al. Balancing curability and unnecessary surgery in the context of computed tomography screening for lung cancer. J Thorac Cardiovasc Surg 2014;147:1619-26.

115.International Early Lung Cancer Action Program Investigators. International Early Lung Cancer Action Program (I-ELCAP) protocol. Available online: https:// www.ielcap.org/protocols

116.Zhang L, Yip R, Jirapatnakul A, et al. Lung cancer screening intervals based on cancer risk. Lung Cancer 2020;149:113-9.

117. Travis WD, Brambilla E, Noguchi M, et al. International association for the study of lung cancer/American thoracic society/European respiratory society international multidisciplinary classification of lung adenocarcinoma. J Thorac Oncol 2011;6:244-85.

118. Travis WD, Brambilla E, Noguchi M, et al. International Association for the Study of Lung Cancer/American
Thoracic Society/European Respiratory Society: international multidisciplinary classification of lung adenocarcinoma: executive summary. Proc Am Thorac Soc 2011;8:381-5.

119. Travis WD, Brambilla E, Burke AP, et al. Introduction to The 2015 World Health Organization Classification of Tumors of the Lung, Pleura, Thymus, and Heart. J Thorac Oncol 2015;10:1240-2.

120. Travis WD, Asamura H, Bankier AA, et al. The IASLC Lung Cancer Staging Project: Proposals for Coding T Categories for Subsolid Nodules and Assessment of Tumor Size in Part-Solid Tumors in the Forthcoming Eighth Edition of the TNM Classification of Lung Cancer. J Thorac Oncol 2016;11:1204-23.

121.Mini Symposium on "Lung Cancer Screening, Opportunistic Evaluation of Findings". IASLC 2019 WCLC World Conference on Lung Cancer; 2019 September 9, 2019; Barcelona, Spain.

122. Shemesh J, Henschke CI, Farooqi A, et al. Frequency of coronary artery calcification on low-dose computed tomography screening for lung cancer. Clin Imaging 2006;30:181-5.

123. Shemesh J, Henschke CI, Shaham D, et al. Ordinal scoring of coronary artery calcifications on low-dose CT scans of the chest is predictive of death from cardiovascular disease. Radiology 2010;257:541-8.

124. de Torres JP, Bastarrika G, Wisnivesky JP, et al. Assessing the relationship between lung cancer risk and emphysema detected on low-dose CT of the chest. Chest 2007;132:1932-8.

125.Zulueta JJ, Wisnivesky JP, Henschke CI, et al. Emphysema scores predict death from COPD and lung cancer. Chest 2012;141:1216-23.

126. Sanchez-Salcedo P, Wilson DO, de-Torres JP, et al. Improving selection criteria for lung cancer screening. The potential role of emphysema. Am J Respir Crit Care Med 2015;191:924-31.

127. International Early Lung Cancer Action Program Investigators. International Early Lung Cancer Action Program protocol. Available online: www.IELCAP.org/ protocols

128. Rami-Porta R, Bolejack V, Crowley J, et al. The IASLC Lung Cancer Staging Project: Proposals for the Revisions of the T Descriptors in the Forthcoming Eighth Edition of the TNM Classification for Lung Cancer. J Thorac Oncol 2015;10:990-1003.

129. Detterbeck FC, Boffa DJ, Kim AW, et al. The Eighth Edition Lung Cancer Stage Classification. Chest 
2017;151:193-203.

130. Goldstraw P, Chansky K, Crowley J, et al. The IASLC Lung Cancer Staging Project: Proposals for Revision of the TNM Stage Groupings in the Forthcoming (Eighth) Edition of the TNM Classification for Lung Cancer. J Thorac Oncol 2016;11:39-51.

131. Nakamura K, Saji H, Nakajima R, et al. A phase III randomized trial of lobectomy versus limited resection for small-sized peripheral non-small cell lung cancer (JCOG0802/WJOG4607L). Jpn J Clin Oncol 2010;40:271-4.

132. CALGB 140503: A phase III randomized trial of lobectomy versus sublobar resection for small $(\leq 2 \mathrm{~cm})$ peripheral non-small cell lung cancer (NCT00499330): National Cancer Institute; Available online: http://www. cancer.gov/about-cancer/treatment/clinical-trials/search/ view? cdrid=555324

133. Suzuki K, Saji H, Aokage K, et al. Comparison of pulmonary segmentectomy and lobectomy: Safety results of a randomized trial. J Thorac Cardiovasc Surg 2019;158:895-907.

134.Altorki NK, Wigle D, Wang X, et al. Mortality and Morbidity of Lobar versus Sub-lobar Resection in CALGB 140503 (ALLIANCE). American Association for Thoacic Surgery (AATS) 98th Annual Meeting; 2018; San Diego, CA.

135.Altorki NK, Yip R, Hanaoka T, et al. Sublobar resection is equivalent to lobectomy for clinical stage 1A lung cancer in solid nodules. J Thorac Cardiovasc Surg 2014;147:754-64.

136. Buckstein M, Yip R, Yankelevitz D, et al. Radiation therapy for stage I lung cancer detected on computed tomography screening: results from the international early lung cancer action program. J Radiat Oncol 2014;3:153-7.

137.Flores R, Nicastri D, Bauer T, et al. The Role of Surgical Mediastinal Resection in CT Screen-Detected Lung Cancer Patients. J Thorac Oncol 2015;10:S242-3.

138. Berlin E, Buckstein M, Yip R, et al. Definitive Radiation for Stage I Lung Cancer in a Screened Population: Results From the I-ELCAP. Int J Radiat Oncol Biol Phys 2019;104:122-6.

139.Schwartz RM, Yip R, Olkin I, et al. Impact of surgery for stage IA non-small-cell lung cancer on patient quality of life. J Community Support Oncol 2016;14:37-44.

140.Schwartz RM, Yip R, Flores RM, et al. The impact of resection method and patient factors on quality of life among stage IA non-small cell lung cancer surgical patients. J Surg Oncol 2017;115:173-80.

141. Schwartz RM, Gorbenko K, Kerath SM, et al. Thoracic surgeon and patient focus groups on decision-making in early-stage lung cancer surgery. Future Oncol 2018;14:151-63.

142.Yip R, Taioli E, Schwartz R, et al. A Review of Quality of Life Measures used in Surgical Outcomes for Stage I Lung Cancers. Cancer Invest 2018;36:296-308.

143. Schwartz RM, Alpert N, Rosenzweig K, et al. Changes in quality of life after surgery or radiotherapy in early-stage lung cancer. J Thorac Dis 2019;11:154-61.

144. Flores R, Taioli E, Yankelevitz DF, et al. Initiative for Early Lung Cancer Research on Treatment: Development of Study Design and Pilot Implementation. J Thorac Oncol 2018;13:946-57. doi: $10.21037 /$ ccts-20-125

Cite this article as: Yip R, Jirapatnakul A, Li M, Yankelevitz DF, Henschke CI. Review of the future of lung cancer screening. Curr Chall Thorac Surg 2022;4:42. 